\title{
Less Developed Countries, Tourism Investments and Local Economic Development*
}

\author{
Rainer Andergassen† Guido Candela
}

September 17, 2009

Department of Economics, University of Bologna, Piazza Scaravilli 2, 40126, Bologna, Italy

\begin{abstract}
The present paper analyzes the forward linkages of a multinational's investment in a resort which kicks off tourism activity in a LDC. We show that, under quite natural assumptions, overnight stays are increasing in the number of tourism related, differentiated goods and services supplied in equilibrium. These goods and services, if supplied by the local community, represent forward linkages of FDI in tourism. We investigate the multinational's incentives to promote, reduce or eliminate these forward linkages and the effectiveness of some policy instruments available to a local government to leverage on the presence of FDI and to stimulate domestic entrepreneurship.
\end{abstract}

Keywords: Tourism FDI; multinationals; economic development; domination strategies; LDC

JEL: L83; F23; O19;

\footnotetext{
*The authors would like to thank Roberto Cellini and Antonello Scorcu for helpful comments.

†Corresponding author. E-mail addresses: rainer.andergassen@unibo.it, guido.candela@unibo.it
} 


\section{Introduction}

Many policy makers view FDI as a way to further the process of wealth creation in the host country and to help to jump-start the development process in less developed countries (LDCs). The implications in terms of economic policy are the creation of a favorable economic environment adapt to attract FDI, consisting, for example, of special tax treatment, exemption from import duties or direct subsidies. Academic literature studies the welfare effects of FDI on both the host and the home country, unveiling potential channels of welfare gains as well as welfare losses ${ }^{1}$. FDI create backward and forward linkages in the host country (Rodriguez-Claire, 1996), that is vertical and horizontal externalities $^{2}$. The negative competitive pressure effects of FDI are countered by productivity gains owing to technology transfer and diffusion, access to knowledge capital and, on the account of learning by doing and learning by observing, skill transfer. Technological upgrade and efficiency gains may also be induced and accelerated by the competitive pressure effects of FDI, which drive inefficient firms in the host country out of the market. Linkage effects on welfare may be positive or negative, depending on the prevalence of positive productivity-efficiency effects and negative crowding out effects of local firms. The host country may also benefit from market access spill-overs, which are due to the knowledge acquisition of international marketing, the access to international distribution markets and the opening up of international markets. In the home country, FDI may lead to productivity increases because of economies of scale and learning curve effects, which counter negative employment effects resulting from the international reallocation of production facilities.

In the present paper we study the effects of a multinational's tourism investment on local economic development $^{3}$. FDI is often considered to be one of the most effective engines for harnessing the development of capital, infrastructure and knowledge and for granting access to global marketing in tourism. Therefore, LDCs often view the attraction of such investments as a leading tourism and economic development strategy but the theoretical and empirical implications of tourism FDI on the host country are not well studied and understood ${ }^{4}$.

FDI, which is mostly concentrated in hotels and restaurants, is most important for emerging tourism economies, while it is relatively less important for developed and mature tourism markets. UNCTAD

\footnotetext{
${ }^{1}$ For a survey see, for example, Markusen (1995), Blomstrom and Kokko (1998), Hanson (2001), Lipsey (2004), Navaretti and Venables (2004).

${ }^{2}$ FDI are often related to imperfection in the technology market, i.e. tacit knowledge, and framed in Dunning's OLI paradigm, that is ownership, location and internalization advantage (see Dunning, 1981, 1993 and Markusen 1995 for a discussion).

${ }^{3}$ In a companion paper by Antonelli and Candela (2008) the relationship between multinationals and the development of a tourism destination is investigated.

${ }^{4}$ The literature on FDI in tourism consists mainly of case studies. See, for example, UNCTAD (2007).
} 
(2007) documents the strong impact of FDI on consumer demand in new destinations. For example, FDI played a major role in the tourism take-off in Tunisia in the 1970s, when investors such as Club Med invented the concept of a beach resort. In a similar vein, in the Dominican Republic, despite governmental investments to start a tourism industry in the 1970s, the real boom did not occur before the massive influx of FDI from the 1980s onwards. UNCTAD (2007) documents also the case of Bhutan and the United Republic of Tanzania where sharp increases in tourist arrival numbers appear to be directly linked to FDI.

FDI raise the policy challenge of taking full advantage of these investments, that is to leverage on the presence of FDI to foster domestic entrepreneurship. These policies consists, for example, in minimizing leakages ${ }^{5}$, in improving the tourism value chain and, more generally, policies aimed at boosting the net value of tourism that have the effect of increasing tourists' length of stay.

The role of FDI in tourism is more nuanced than it is in some other sectors of the economy, and it is valued because of the kick off to the tourism industry which it can provide, but it is also feared for its impact on economic and cultural independence, and its potential damage to the communities and the environment (UNCTAD, 2007). The country faces the risk of foreign domination, such as enclaves and other strategies where the multinational limits the benefits for the host country and where the country is therefore unable to capitalize on such investments. This is the case, for example, of Punta Cana, a Dominican Republic island, where transnational corporations built small enclaves equipped with their own essential services such as power, waste and water management and access modes (UNCTAD, 2007). Brohman (1996) argues that foreign domination and external dependency often seriously reduce tourism's potential for generating broadly based growth and takes the case of the Caribbean as an example, where a spatial polarization within both the modern tourism industry, based on resort enclaves in the most desirable coastal locations, and the older agricultural-based economy emerged. Battilani (2002) documents the investment strategy of a pool of entrepreneurs led by Karim Aga Khan IV in the tourism untouched Costa Smeralda in Sardegna (Italy) during the 1960's, who held the development of tourism and in general of the economic region under tight control acquiring a monopoly position on the potentially usable land and controlling the administration and the development of the region.

We consider the case of a multinational who builds and owns a resort in a new destination in a LDC and study the multinational's incentives to make the local community participant of the tourism

\footnotetext{
${ }^{5}$ Leakages in the tourism sense, that is negative national balance-of-payment effects such as the imports of goods and the repatriation of profits.
} 
development. We show that under quite natural assumptions, tourists' overnight stays are increasing in the degree of diversification of tourism related products and services. Therefore, the multinational's profits are strictly related to the variety of tourism related goods and services and consequently the multinational has an incentive to control its supply. In particular, the multinational faces two options. On the one hand the multinational can try to control the variety of goods and services supplied by local firms and on the other hand it can create an enclave and supply directly the preferred variety. In the former case, in which tourism is based on culturally authentic goods and services, we assume that each differentiated product is produced by a single firm and hence the number of tourism related products supplied is a proxy for the local economic development induced by the multinational's investments. In the latter case, in which tourism is based on a fake but authentically staged local variety, the local community does not benefit from the tourism activity.

Our analysis focuses on these forward linkages of FDI, that is the creation of new markets in a LDC through the tourism product, and we neglect backward linkages established by resorts, that is suppliers of intermediate consumption goods.

We consider a small open subsistence economy. All firms are price taker on the international tourism market. We assume that the production of differentiated products necessitates a fixed set up cost, which accounts for the shadow price of land and the cost of turning unproductive land into productive land ${ }^{6}$. Available and potentially usable land is in perfect elastic supply up to a fixed level and its supply is assumed to be perfectly inelastic afterwards.

In Section 2 we characterize the representative consumer's demand function consisting of overnight stays, consumption of differentiated, tourism related products and non-tourism related products. We show how the multinational's profits from overnight stays and the supply of differentiated products are related and that, from the multinational's viewpoint, there exists an optimal degree of product diversification. Therefore, if tourism products are supplied by the local community, where the local variety is determined by a free entry condition, then there may be too many or too few products from the multinational's viewpoint. As a consequence, the multinational may have an incentive to either foster or curb local economic development or eventually to substitute the local authentic variety of tourism goods with an authentically staged variety supplied by the multinational. We show that, for the multinational, subsidizing local development, that is subsidizing the local firm's set up cost, is

\footnotetext{
${ }^{6}$ As argued above, tourism related FDI is most important for new destinations and in emerging economies. Once the destination develops and becomes mature, the relative importance of FDI diminishes. Therefore, in this context crowding out effects of FDI are negligible and we accordingly assume that the shadow price of land, which accounts for alternative uses of land, is unaffected by the multinational's investment strategy.
} 
never optimal. On the other hand we show that the multinational may curb local development, by buying all the available land and reselling part of it at a positive margin. We further show that from the multinational's profits viewpoint this is equivalent to creating an enclave where the local variety is authentically staged by the multinational. The difference between these two strategies is that in the former case the variety is truly authentic, while in the second case it is an authentically staged variety. We call these two strategies domination strategies since the multinational controls tourism development in the host country ${ }^{7}$.

In Section 3 we discuss the effectiveness of policy instruments available to a local government who's aim is to maximize local economic development. We show that taxing the multinational's activity as well as subsidizing directly local economic development by subsidizing the firm's set up cost may well be counterproductive since it increases the multinational's incentives to engage in a domination strategy. On the other hand, we show that infrastructural investments aimed at increasing the potentially available land may be beneficial since they reduce the multinational's gains from domination strategies.

Section 4 concludes. All proofs are in the Appendix.

\section{The model}

In the first part of this section we describe the representative consumer's tourism demand function. We then characterize the supply side. We calculate the equilibrium number of differentiated, locally supplied, tourism related products which is determined by a free entry condition and the optimal supply of tourism related products from the multinational's point of view. Finally, we characterize the multinational's optimal domination strategy.

\subsection{Tourism demand}

In this Section we characterize tourism demand consisting of nights spent in a destination, that is overnight stays in the resort, and consumption of tourism related products. We consider a representative consumer endowed with a constant elasticity of substitution (CES) utility function exhibiting

\footnotetext{
${ }^{7} \mathrm{~A}$ further difference between the two strategies is that in the case of an enclave the production process is a vertically integrated one, where the multinational acts as a coordinator of the supply, while in the case of a authentic local variety the lack of coordination among producers may lead to a suboptimal outcome from the destination's viewpoint.
} 
Dixit and Stiglitz (1977) love of variety preferences for differentiated tourism related goods

$$
U\left(y, h, x_{1}, \ldots, x_{i}, \ldots, x_{n}\right)=\left\{y^{\beta}+\left[h^{\gamma}+\left(\sum_{i=1}^{n} x_{i}^{\alpha}\right)^{\frac{\gamma}{\alpha}}\right]^{\frac{\beta}{\gamma}}\right\}^{\frac{1}{\beta}}
$$

where $y$ indicates a composite, non-touristic good, $h$ indicates overnight stays and $x_{i}$, for $i=1, \ldots, n$, indicates differentiated tourism related goods. We assume that at least one variety has to be offered such that tourism is viable, i.e. $n \geq 1$. We call $T$ the tourism product, consisting of overnight stays (h) and differentiated tourism related products $\left(\left\{x_{i}\right\}_{i=1}^{n}\right)$, i.e. $T=\left(h,\left\{x_{i}\right\}_{i=1}^{n}\right)$.

The representative consumer faces the budget constraint

$$
y+p_{h} h+\sum_{i=1}^{n} p_{i} x_{i}=R
$$

where $R$ is his income, $p_{h}$ is the price of a single overnight stay, $p_{i}$ the price of $x_{i}$ and where the price of the non-touristic good $y$ has been normalized to 1 .

Throughout the paper we assume the following.

Assumption 1 (i) $0<\beta<1$, (ii) $-\infty<\gamma<0$, (iii) $0<\alpha<1$, (iv) $\alpha>\beta$.

Assumption 1 (i) implies that the non-touristic good $y$ and the tourism product $T$ are gross substitutes; for $\beta \rightarrow 1$ they are perfect substitutes. Assumption 1 (ii) implies that overnight stays and tourism related products are gross complements, where for $\gamma \rightarrow-\infty$ they are perfect complements. Assumption 1 (iii) implies that goods/services $x_{i}, i=1, \ldots, n$, are gross substitutes. Assumption 1 (iv) states that the gross substitutability of tourism related goods/services is greater than the gross substitutability between the non-touristic goods $(y)$ and the tourism product $(T)$. We define $\lambda_{\beta}=\frac{\beta}{1-\beta} \in(0, \infty)$, $\lambda_{\gamma}=\frac{\gamma}{1-\gamma} \in(-1,0), \lambda_{\alpha}=\frac{1-\alpha}{\alpha} \in(0, \infty)$. We assume symmetry on the supply side where $p_{i}=p$ and therefore in equilibrium $x_{i}=x$, for $i=1, \ldots, n$.

Assumption 2 Prices $p$ and $p_{h}$ and income $R$ are such that the following inequalities are satisfied: (i)

$$
p_{h}\left[1+\left(\frac{p}{p_{h}}\right)^{-\lambda_{\gamma}}\right]\left\{1+p_{h}^{\lambda_{\beta}}\left[1+\left(\frac{p}{p_{h}}\right)^{-\lambda_{\gamma}}\right]^{-\frac{\lambda_{\beta}}{\lambda_{\gamma}}}\right\}>R \frac{z}{p_{h}}>p_{h}\left(1+p_{h}^{\lambda_{\beta}}\right)
$$

(ii) $p_{h}>p$ and

$$
\frac{p}{p_{h}}<\left(-\frac{\lambda_{\gamma}}{\lambda_{\beta}}\right)^{-\frac{1}{\lambda_{\gamma}}}
$$


Assumption 2 (i) guarantees that the below defined profit maximization problem of the multinational has an internal maximum. In particular, if the first inequality is not satisfied then the preferred number of tourism related products to be supplied, if the multinational does not engage in a domination strategy, is the minimum possible, i.e. $n=1$. If the second inequality is not satisfied, then the solution diverges to infinity. Assumption 2 (ii) states that the price of an overnight stay is larger than the price of tourism related products and further that the substitutability between the non-touristic good $(y)$ and the tourism product $(T)$ is sufficiently low.

Maximizing (1) subject to the budget constraint (2) we obtain the following result.

Lemma 1 The optimal number of overnight stays is strictly increasing in $n$, i.e. $h^{\prime}(n)>0$; (ii) the demand of differentiated products is strictly deceasing in $n$, i.e. $x^{\prime}(n)<0$; (iii) the demand of the non-touristic good is strictly decreasing in $n$, i.e. $y^{\prime}(n)<0$.

Lemma 1 relates the degree of differentiation of the tourism product $(n)$ to the number of overnight stays. In particular, it states that the more "sophisticated" is the tourism product, that is the greater is $n$, the more days tourists spend in a given destination and hence the greater is their expenditure on overnight stays. A sufficient condition for this result to hold is that either overnight stays and tourism related products are gross complements, or that the non-touristic good and the tourism product $T$ are gross substitutes $^{8}$. The assumption about the gross substitutability of non-touristic goods with the tourism product $T$ implies that an increase in $n$ decreases demand for and expenditure on non-touristic goods and therefore increases the consumers' expenditure on touristic goods. Moreover, Assumptions 1 (i) and (iv) guarantee that, as $n$ increases, the demand of $x$ decreases ( $h$ increases) at a faster pace than if $\beta=0$. The assumption of greater substitutability between tourism related goods than between the non-touristic good and the tourism product $T$ leads consumers to spend relatively more on overnight stays and relatively less on tourism related products as $n$ increases.

\subsection{Supply side}

For simplicity's sake we assume that available and potentially usable land is in perfect elastic supply up to a fixed level $N$ and its supply is assumed to be perfectly inelastic afterwards. The firm's set up cost is $c$.

\footnotetext{
${ }^{8}$ If $\beta=0$, that is the non-touristic good $y$ and the tourism product $T$ are independent, and $\gamma=0$, that is if overnight stays and the differentiated tourism goods are independent, then the demand for overnight stays $h$ is independent of $n$.
} 
In this section we focus on the case where differentiated products and services are produced by local firms, that is the case of a culturally authentic local variety, and show how the multinational's interests may be conflicting with those of a local government whose aim we assume to be the promotion of local economic development.

We assume that each differentiated product/service is produced by a single firm and that production cost are nil ${ }^{9}$. The free entry condition for local firms is

$$
p x\left(n^{L}\right)=c
$$

which implicitly defines the number of firms operating in the tourism sector $n^{L}$. Notice that $n^{L} \geq 1$ if $c$ is sufficiently low, that uniqueness is guaranteed by the fact that $x(n)$ is strictly decreasing in $n$ and that $n^{L}$ is decreasing in $\frac{c}{p}$. We also assume that $N \geq n^{L}$.

In order to calculate the optimal degree of tourism product differentiation from the multinational's viewpoint, we calculate the optimal resort capacity in terms of overnight stays. Assuming a quadratic cost function for the production of overnight stays $z \frac{1}{2} h^{2}$, the multinational's profits are ${ }^{10}$

$$
\pi=p_{h} h-z \frac{1}{2} h^{2}
$$

Since overnight stays depend on $n$ we can characterize $n^{M}$, which is the optimal $n$ from the multinational's viewpoint. First order condition yields $n^{M}$, which is implicitly defined by $h\left(n^{M}\right)=\frac{p_{h}}{z}$. Assumption 2 together with $h^{\prime}(n)>0$ guarantee that there exists a unique $n^{M}$ which is strictly increasing in $\frac{p_{h}}{z}$.

The following Lemma establishes the relationship between $n^{M}$ and $n^{L}$.

Lemma 2 Given $z, p$ and $p_{h}$, let $\left(c^{e}, n^{e}\right)$ be the unique solution to

$$
\left\{\begin{array}{l}
x\left(n^{e}\right)=\frac{c^{e}}{p} \\
h\left(n^{e}\right)=\frac{p_{h}}{z}
\end{array}\right.
$$

then: (i) for $c=c^{e}, n^{M}=n^{L}=n^{e}$; (ii) for $c>c^{e}, n^{M}-n^{L}>0$ and where the difference is increasing in $c$; (iii) $c<c^{e}, n^{L}-n^{M}>0$ and where the difference is decreasing in $c$.

\footnotetext{
${ }^{9}$ Assuming in addition to fixed setup costs constant marginal costs of production would not change the qualitative results.

${ }^{10}$ We normalize the multinational's fixed costs (or set up costs), to zero, and consider only operating revenues and costs. Taking entry cost into account would affect the multinational's decision to enter the destination or not.
} 
According to Lemma 2, depending on the value $c$ takes, the local community's interest, that is to promote local economic development, and the multinational's, which lies in the exploitation of profit opportunities, may either be conflicting or aligned. In particular, there exists a critical cost level $c^{e}$ above which the degree of product differentiation provided by the local community is too low compared with the multinational's optimal level and below which it is too large. These conflicting interests raise the problem that the multinational may try to control the provision of tourism related products, thereby sustaining or hampering local economic development.

\subsection{The multinational's domination strategy}

We consider the case where (a) the multinational finances local development by subsidizing local firms' set up cost which becomes $c+s, s \in(-c, 0)$ and (b) the multinational buys all the available land $N$ at the price $c$, becoming in this way a monopolist, and resells it at a cost $c+s, s \in(0, \infty)$. The former case may be relevant if the local community supplies too few differentiated products compared with the multinational's optimal level, while the latter case addresses the issue of an excess-differentiation. Later on we argue that, from the multinational's viewpoint, the latter situation is equivalent to creating an enclave, the only difference being that if tourism related products are produced by local firms, then they are culturally authentic goods while in the case of an enclave they consist of fake, but authentically staged products.

Given the value of $s$, if differentiated goods and services are produced by local firms, then the free entry condition (3) reads

$$
p x(n)=c+s
$$

which implicitly defines the equilibrium number of local firms $n(s)$, with $n^{\prime}(s)<0$. In the present context, because of (5), choosing $s$ is equivalent to choosing $n$. Therefore, the multinational's problem is to choose $n$ which maximizes profits from its core business, that is profits from overnight stays, and profits or losses related to the direct or indirect control of the degree of product differentiation.

Consider first the multinational's incentives to subsidizes local development. For this purpose we have to compare the multinational's profits if it subsidizes local development, that is $\max _{s \leq 0} \widetilde{\pi}(s)$, where $\widetilde{\pi}(s)=\pi(n(s))+s n(s)$, with those if it does not intervene, i.e. $\pi\left(n^{L}\right)$. The following proposition can be proved.

Proposition 1 Subsidizing local development is never optimal. 
The intuition for this result is as follows. Because of the assumption of greater gross substitutability between the single tourism related goods than between the non-touristic good and the tourism product $T$ (Assumption 1 (iv)), the demand $x(n)$ decreases at a faster pace as $n$ increases. As a consequence, it is more costly to increase $n$ and the gains from an increase in $n$ are outweighed by the cost of doing so.

Notice that a consequence of Proposition 1 is that, if an enclave is optimal, then the degree of product differentiation will never be larger than the one provided by the local community. The intuition for this is as follows. As argued below, the multinational's profits are the same if it creates an enclave or if it buys all the land and resells it at a margin or discount to local producers. In subsidizing local firms, the multinational operates as if it buys all the requested land for a given, announced $s<0$ and resells it to local firms at a price $c+s$. Since subsidizing is never optimal, it follows that it is never optimal to buy more than the required amount of land for an announced $s<0$ and to resell it at a discounted price $c+s$. Therefore, if subsidizing the set up cost is never optimal, then it is never optimal to create an enclave with more differentiated products.

Let us consider the case where the multinational hampers local development or creates an enclave. The multinational acquires a monopoly position by buying all the available land $N$ and then resells it at an increased price $c+s, s \in(0, \infty)$. Since Proposition 1 states that $s \in(-c, 0)$ is never optimal, the multinational's problem can be written as

$$
\max _{s} \Pi(s)=\pi(n(s))+(c+s) n(s)-c N
$$

or using (5), equivalently

$$
\max _{n} \Pi(n)=\pi(n)+p x(n) n-c N
$$

The first element of (6) and/or (7) are profits related to the firm's core business, while the remaining two elements are associated with the buying and selling of land (i.e. domination strategy). Notice that, in the case of an enclave, the multinational's profits are given by $(7)^{11}$. The reason for this is as follows. To create an enclave the multinational has to acquire a monopoly position, that is to eliminate all potential competition from local producers (third element in (7)). Selling the authentically staged variety to the tourists yields the multinational proceeds $p x(n) n$, the second element in ( 7$)$, while the

\footnotetext{
${ }^{11}$ We implicitly assume that the tourist's demand is independent of whether tourism related goods are authentic or fake, but authentically staged, and that the production costs are the same. The qualitative results stated in the paper hold also if this assumption is to be relaxed, that is if tourists prefer one over the other, and/or if the production costs differ.
} 
first element in (7) represents the multinational's proceeds from the core business.

In choosing $n$, or equivalently $s$, the multinational has to trade off gains/costs from its core business against gains / costs related to the domination strategy. We first show that a unique $n^{*}=\arg \max _{n} \Pi(n)$ to the problem (7) exists and, using (5), we characterize the corresponding $s^{*}$. We then derive conditions such that restraining local development, or equivalently creating an enclave, is optimal.

Lemma 3 There exists a unique $n^{\star}$, with $\frac{\partial}{\partial z} n^{*}<0$.

Since $s$ and $n$ are related through (5), Lemma 3 states that there exists a unique $s^{*}$ which maximizes the multinational's profits. Moreover, it states that $s^{*}$ is increasing in the cost parameter $z$. The reason for this result is that the larger are the costs associated with its core business, the more profitable becomes the domination strategy and hence it is optimal to increase $s^{*}$. Moreover, from (6) and Lemma 3 we observe that $n^{*}$ is independent of $c$ and therefore, by using (5), we have that $s^{*}$ is decreasing in c. The following result characterizes $s^{*}$.

Lemma 4 For $c \leq c^{e}$, and also for some values of $c>c^{e}, s^{\star}>0$.

Lemma 4 states that the multinational may have an incentive to engage in a domination strategy even though this worsens its core business. The intuition for this result is the following. For $c<c^{e}$, where $n^{L}>n^{M}$, a positive $s$ leads to gains in the core business as well as gains from the domination strategy. Consequently, $s^{*}>0$ is optimal. If $c=c^{e}$, then from Lemma 2 we know that the free entry condition leads to an equilibrium with maximizes the multinational's profits in the absence of domination activity, i.e. $n^{L}=n^{M}=n^{e}$. Because of the local concavity of the profit function $\pi$, increasing $s$ leads to a second order loss in its core business and to a first order gain in its domination activity. As a consequence, engaging in a domination strategy is optimal for $c=c^{e}$. By continuity the first order gain from a domination strategy is larger than the second order loss in the core business also for some values of $c>c^{e}$, where $n^{L}<n^{M}$.

Summing up, we observe that if $n^{L} \geq n^{M}$, then $s^{*}>0$. Moreover, the larger the difference between $n^{L}$ and $n^{M}$, i.e. the lower is $c$ (see Lemma 2), the larger is $s^{*}$ and the larger are the gains associated with the domination strategy. For some $n^{L}<n^{M}, s^{*}>0$ is still optimal and increasing the difference between $n^{L}$ and $n^{M}$, that is increasing $c$ (see Lemma 2), decreases $s^{*}$.

The next proposition characterizes the multinational's incentives to curb local development, or equivalently to create an enclave. 
Proposition 2 There exists a threshold $\bar{N}$ such that for all $N>\bar{N}$ engaging in a domination strategy $\left(s^{*}>0\right)$ is never optimal, while for $N<\bar{N}$ engaging in a domination strategy is optimal. $\bar{N}$ is decreasing in $c$ and increasing in $z$.

Proposition 2 states that the lower is the availability of potentially useful land $(N)$ and/or the lower are the set up costs of a local variety $(c)$, the more likely it is that the multinational engages in a dominating strategy. The intuition for this result is that the lower is $N$ and/or $c$, the cheaper it is for the multinational to acquire a monopoly position and therefore to control local economic development. On the other hand, the larger is $z$, the larger are the costs associated with its core business and therefore the more profitable becomes the domination strategy.

\section{Economic policy}

In this section we address the issue of the effectiveness of some economic policy options available to a local government who aims at leveraging on the multinational's investment. In particular, we consider policies which consist in trying to reduce the enclave risk and to maximize local economic development. We analyze the consequences of (i) subsidizing the set up cost $c$, (ii) taxing multinational's activity (in particular its inputs) and (iii) increasing the potentially available land $N$. In case (i) the government tries directly to foster local development, subsidizing the local firms' set up costs. In case (ii) we consider taxes on the multinational's purchases and activities (for example, taxing the multinational's imports), which we model by considering in the above analysis $\bar{z}$ instead of $z$, where $\bar{z}=z(1+t)$ and $t>0$ is the marginal, constant, tax rate ${ }^{12}$. The government may try in this way to cash in on the multinational's activity. In case (iii) the government invests in infrastructure, thereby increasing the amount of land which may be put to productive use (at unit cost $c$ ).

Case (i). We have to distinguish between the case where engaging in a dominating strategy is and is not optimal. Consider first the case where the multinational does not engage in a dominating strategy $(N>\bar{N})$. Tourism products are supplied by local firms and subsidizing the firm's set up cost increases the degree of product differentiation and fosters local economic development. But since $\bar{N}$ is decreasing in $c$, a too strong subsidy leads the multinational to take a dominating position. Once $N<\bar{N}$, Lemma 3 states that an optimal $n^{*}$ exists, which is independent of $c$ and hence the effects of further decreases in $c$ are offset by increases in $s^{*} .^{13}$ Therefore, for $N<\bar{N}$, this policy instrument is

\footnotetext{
${ }^{12}$ The range of variation of the tax rate $t$ is restricted by Assumption 2.

${ }^{13}$ We implicitly assume that the government cannot tie in the granting of the subsidy with a no-sale clause of the land.
} 
ineffective. Consequently, economic policy aimed at directly sustaining economic development may be partly, completely or more than offset by a multinational's domination strategy. Subsidies may have the perverse effect of curbing instead of sustaining economic development. To see this, consider the case of $N$ being larger than, but very close to $\bar{N}$. A decrease in $c$ may lead to $N<\bar{N}$ and therefore to a domination position $\left(s^{*}>0\right)$, which may well lead to an overall reduction in $n$.

Case (ii). From Lemma 2, considering $\bar{z}$ instead of $z$, we observe that $c^{e}$ is increasing in $\bar{z}$, where, for $c>c^{e}, n^{M}-n^{L}>0$ with $n^{M}-n^{L}$ decreasing in $\bar{z}$, while for $c<c^{e}, n^{L}-n^{M}>0$, with $n^{L}-n^{M}$ increasing in $\bar{z} \cdot{ }^{14}$ Lemma 4 and the positive relationship between $\bar{N}$ and $\bar{z}$ implies that the larger are taxes, that is the larger is $\bar{z}$, the greater are the multinational's incentives to engage in a domination strategy. Moreover, from Lemma 3 we know that $s^{*}$ is increasing in $\bar{z}$. The intuition for these results is as follows. Increasing taxes $t$ increases the multinational's costs associated with its core business and hence a domination strategy becomes more attractive. As a consequence, as long as $N$ is larger than $\bar{N}$, small taxes on the multinational's activity lead to tax yields and do not induce the multinational to engage in a domination strategy. But if taxes are too large and $N<\bar{N}$, then the multinational engages in a domination strategy, where larger taxes imply a stronger domination position and therefore a stronger restrain on economic development. Therefore, the government has to trade off welfare benefits from tax yields against their costs owing to a reduced economic development. If the government wants to promote economic development through tourism, a complete or partial tax exemption for the multinational may be preferable.

Case (iii). From the analysis above it follows that increasing $N$, by increasing the multinational's costs of engaging in a domination strategy, decreases the profitability of such investments. This policy unambiguously decreases the multinational's incentives to engage in a domination strategy and therefore increases the possibility for the local community to benefit from tourism FDI. It remains an open question how to finance such investments. The government may well try to pursue a mixed strategy, that is financing infrastructural investments through taxes on the multinational's activity, under the constraint that $N>\bar{N}$. Moreover, the government may also try to tie in tourism FDI with infrastructural investments, that is to approve FDI only if these are associated with infrastructural investments aimed at avoiding domination strategies.

\footnotetext{
${ }^{14}$ To see this consider the case where $c=c_{e}$. Given that an increase (decrease) in $\bar{z}$ leads to an increase (decrease) in $c^{e}$ we are, for a constant value of $c$, in case Lemma 2 (iii) (in case Lemma 2 (ii)) where, since $n^{L}$ is independent of $\bar{z}$, $n^{L}=n^{e}>n^{M}\left(n^{L}=n^{e}<n^{M}\right)$.
} 


\section{Conclusion}

The present paper analyzed the forward linkages of a multinational's investment in a resort that kicks off the tourism sector in a LDC. We showed that, under Assumption 1, overnight stays are increasing in the number of differentiated tourism related goods and services, which, if supplied by local firms, is a proxy for the economic development induced by FDI. We therefore argued that the multinational has an incentive to control the variety of these goods supplied in equilibrium. We showed that it is never optimal for a multinational to subsidizes local development and showed that the multinational may engage in a domination strategy aimed at reducing or even eliminating the host country's benefits from the tourism activity. This result is in keeping with the historical evidence that the presence of enclaves is associated with a low degree of differentiation of tourism related products. We analyzed the effectiveness of some policy instruments available to a local government whose goal is to leverage on the multinational's investments and we showed that domination strategies can be avoided by engaging in infrastructural investments designed to increase the potentially available land or by reducing taxes on multinationals acquisitions and expenses. This latter point complements the traditional argument in favor of tax reductions or exemptions for FDI based on the presumption that the supply of international capital is elastic relative to domestic capital (see, for example, Hanson, 2001). Subsidizing directly local development may be counterproductive since it increases the multinational's incentives to adopt a domination strategy.

These policy conclusions are in keeping with some policies undertaken by governments of LDCs. Endo (2006) reports that many LDCs grant the multinational duty free imports. Even though this increases leakages, according to our analysis it decreases the multinational's incentives to engage in domination strategies and therefore it increases the possibility for the host country to leverage on FDI in the tourism sector. UNCTAD (2007) documents that the majority of financial incentives to attract tourism related FDI consists in tax incentives (tax rebates) and duty-free imports of inputs. Battilani (2001) documents that starting from 1978 the government has accompanied the strong influx of FDI in some small, uninhabited islands of the Maldives with infrastructural investments aimed at increasing the tourists' mobility. According to our analysis, these investments increased the multinationals' costs of creating enclaves and therefore it increased the chances for the host country to benefit from the tourism activity. In a similar vein, tourism in Punta Cana in the Dominican Republic, which was initially confined to small enclaves, took off only after investments in resorts by Club Med where tied to the construction of a new local international airport (see UNCTAD, 2007), opening up tourism also 
in other areas and paving the way for the region's economic development.

\section{References}

[1] Antonelli, L. and G. Candela (2008). Multinationals and the development of a tourism destination, mimeo.

[2] Battilani, P. (2001). Vacanze di pochi vacanze di tutti, Il Mulino, Bologna.

[3] Battilani, P. (2002). Rimini and Costa Smeralda: how social values shape recreational sites. In S.C. Anderson, B. Tabb (Ed.), Water Leisure and Culture, Oxford, Berg Publishers.

[4] Blomstrom, M. and A. Kokko (1998). Multinational Corporations and Spillovers, Journal of Economic Surveys, 12, 1 - 31.

[5] Brohman, J. (1996). New directions in tourism for third world development, Annals of Tourism Research, 23, 48-70.

[6] Dixit, A. K., J. E. Stiglitz (1977). Monopolistic competition and Optimum Product Diversity, American Economic Review, 67, 297-308.

[7] Dunning, J.H. (1981). International Production and the Multinational Enterprise. London, George, Allen and Unwin.

[8] Dunning, J.H. (1993). The Globalization of Business. London, Routledge.

[9] Endo, K. (2006). Foreign direct investment in tourism - flows and volumes, Tourism Management, $27,600-614$.

[10] Hanson, G.H. (2001). Should Countries Promote Foreign Direct Investment? G-24 Discussion Paper Series, United Nations.

[11] Lipsey, R.E. (2004). Home and Host Country Effects of FDI. In R.E. Baldwin and L.A. Winters, Eds., Challenges to Globalization, p. 333-379, University of Chicago Press, Chicago.

[12] Markusen, J.R. (1995). The Boundaries of Multinational Enterprises and the Theory of International Trade, Journal of Economic Perspectives, 9, 169-189. 
[13] Navaretti, B.G. and A.J. Venables (2004). Multinational Firms in the World Economy. Princeton University Press, Princeton.

[14] Rodriguez-Clare, A. (1996). Multinationals, Linkages and Economic Development, American Economic Review, 86, 852-73.

[15] UNCTAD (2007). FDI in Tourism: The development dimension, New York and Geneva: United Nations.

\section{Appendix}

Proof. [Proof of Lemma 1] Using Lagrange, the first order conditions read:

$$
\begin{gathered}
\left\{y^{\beta}+\left[h^{\gamma}+\left(\sum_{i=1}^{n} x_{i}^{\alpha}\right)^{\frac{\gamma}{\alpha}}\right]^{\frac{\beta}{\gamma}}\right\}^{\frac{1}{\beta}-1} y^{\beta-1}=\lambda \\
\left\{y^{\beta}+\left[h^{\gamma}+\left(\sum_{i=1}^{n} x_{i}^{\alpha}\right)^{\frac{\gamma}{\alpha}}\right]^{\frac{\beta}{\gamma}}\left[h^{\gamma}+\left(\sum_{i=1}^{n} x_{i}^{\alpha}\right)^{\frac{1}{\alpha}}\right]^{\frac{\beta}{\gamma}-1} h^{\gamma-1}=\lambda p_{h}\right. \\
\left\{y^{\beta}+\left[h^{\gamma}+\left(\sum_{i=1}^{n} x_{i}^{\alpha}\right)^{\frac{\gamma}{\alpha}}\right]^{\frac{\beta}{\gamma}}\right\}^{\frac{1}{\beta}-1}\left[h^{\gamma}+\left(\sum_{i=1}^{n} x_{i}^{\alpha}\right)^{\frac{\gamma}{\alpha}}\right]^{\frac{\beta}{\gamma}-1}\left(\sum_{i=1}^{n} x_{i}^{\alpha}\right)^{\frac{\gamma}{\alpha}-1} x_{i}^{\alpha-1}=\lambda p_{i},
\end{gathered}
$$

for $i=1, \ldots, n$ where $\lambda$ is the Lagrange multiplier.

Using the assumption that all local firms are symmetric we have $p_{i}=p$ and hence obtain $x_{i}=x$, for each $i=1, \ldots, n$. From (9) and (10) we obtain

$$
x=h\left(\frac{p}{p_{h}} n^{1-\frac{\gamma}{\alpha}}\right)^{\frac{1}{\gamma-1}}
$$

while from (9) and (8) we obtain

$$
p_{h}=\frac{\left(h^{\gamma}+n^{\frac{\gamma}{\alpha}} x^{\gamma}\right)^{\frac{\beta}{\gamma}-1} h^{\gamma-1}}{y^{\beta-1}}
$$

which, using (11), reads as

$$
y=h p_{h}^{\frac{1}{1-\beta}}\left[1+n^{\frac{\gamma}{1-\gamma} \frac{1-\alpha}{\alpha}}\left(\frac{p}{p_{h}}\right)^{\frac{\gamma}{\gamma-1}}\right]^{\left(\frac{\beta}{\gamma}-1\right) \frac{1}{\beta-1}}
$$


Finally, we calculate $h$ substituting (11) and (12) into the budget constraint (2)

$$
h=\frac{R}{k(n)}
$$

where

$$
k(n)=p_{h}\left[1+n^{\frac{\gamma}{1-\gamma} \frac{1-\alpha}{\alpha}}\left(\frac{p}{p_{h}}\right)^{\frac{\gamma}{\gamma-1}}\right]\left\{p_{h}^{\frac{\beta}{1-\beta}}\left[1+n^{\frac{\gamma}{1-\gamma} \frac{1-\alpha}{\alpha}}\left(\frac{p}{p_{h}}\right)^{\frac{\gamma}{\gamma-1}}\right]^{-\frac{\beta}{1-\beta} \frac{1-\gamma}{\gamma}}+1\right\} .
$$

We can rewrite (14) as

$$
k(n)=p_{h}\left[1+n^{\lambda_{\gamma} \lambda_{\alpha}}\left(\frac{p}{p_{h}}\right)^{-\lambda_{\gamma}}\right]\left\{1+p_{h}^{\lambda_{\beta}}\left[1+n^{\lambda_{\gamma} \lambda_{\alpha}}\left(\frac{p}{p_{h}}\right)^{-\lambda_{\gamma}}\right]^{-\frac{\lambda_{\beta}}{\lambda_{\gamma}}}\right\}
$$

with

$$
k^{\prime}(n)=p_{h} \lambda_{\gamma} \lambda_{\alpha} n^{\lambda_{\gamma} \lambda_{\alpha}-1}\left(\frac{p}{p_{h}}\right)^{-\lambda_{\gamma}}\left\{1+\frac{\lambda_{\gamma}-\lambda_{\beta}}{\lambda_{\gamma}} p_{h}^{\lambda_{\beta}}\left[1+n^{\lambda_{\gamma} \lambda_{\alpha}}\left(\frac{p}{p_{h}}\right)^{-\lambda_{\gamma}}\right]^{-\frac{\lambda_{\beta}}{\lambda_{\gamma}}}\right\}
$$

Using Assumption 1, which implies that $\lambda_{\beta}>0,-1<\lambda_{\gamma}<0$ and that $\lambda_{\alpha}>0$, we have that $k^{\prime}(n)<0$, and thus $h(n)$ is strictly increasing in $n$. Moreover we can write

$$
x=\frac{R}{p\left[n^{-\lambda_{\gamma} \lambda_{\alpha}}\left(\frac{p}{p_{h}}\right)^{\lambda_{\gamma}}+1\right]} \frac{1}{n+p_{h}^{\lambda_{\beta}}\left[n^{-\frac{\lambda_{\gamma}}{\lambda_{\beta}}}+n^{\lambda_{\gamma} \lambda_{\alpha}-\frac{\lambda_{\gamma}}{\lambda_{\beta}}}\left(\frac{p}{p_{h}}\right)^{-\lambda_{\gamma}}\right]^{-\frac{\lambda_{\beta}}{\lambda_{\gamma}}}}
$$

which, since Assumption 1 (iv) implies that $\lambda_{\alpha} \lambda_{\beta}<1$, is strictly decreasing in $n$, and

$$
y=R \frac{1}{1+p_{h}^{-\lambda_{\beta}}\left[1+n^{\lambda_{\gamma} \lambda_{\alpha}}\left(\frac{p}{p_{h}}\right)^{-\lambda_{\gamma}}\right]^{\frac{\lambda_{\beta}}{\lambda_{\gamma}}}}
$$

which is decreasing in $n$.

Proof. [Proof of Lemma 2] The result follows from system (4). An intuitive proof is as follows. Suppose we are initially in the equilibrium $c=c^{e}$, where $n^{M}=n^{L}=n^{e}$. An increase in $c$ leads to a reduction in $n^{L}$ while $n^{M}$ remains unaffected. Hence, $n^{M}=n^{e}>n^{L}$. A decrease in $c$ leads to an increase in $n^{L}$ and therefore $n^{M}=n^{e}<n^{L}$. 
Proof. [Proof of Proposition 1] We establish the result by showing that $\frac{\partial}{\partial n} \widetilde{\pi}(n)<0$, for each $s<0$. Using (5), the profit function $\widetilde{\pi}$ reads

$$
\widetilde{\pi}(n)=p_{h} h(n)-\frac{1}{2} z h(n)^{2}+[p x(n)-c] n
$$

We rewrite the relationship between $x(n)$ and $h(n)$ in (11) as follows

$$
x(n)=h(n) n^{\lambda_{\gamma} \lambda_{\alpha}-1}\left(\frac{p}{p_{h}}\right)^{-\lambda_{\gamma}-1}
$$

which allows us to write the multinational's profits as

$$
\frac{\tilde{\pi}(n)}{p_{h}}=h(n)\left[1-\frac{1}{2} \frac{z}{p_{h}} h(n)+n^{\lambda_{\gamma} \lambda_{\alpha}}\left(\frac{p}{p_{h}}\right)^{-\lambda_{\gamma}}\right]-\frac{c}{p_{h}} n
$$

Taking the first derivative with respect to $n$ we obtain

$$
\frac{1}{p_{h}} \frac{\partial}{\partial n} \widetilde{\pi}(n)=h^{\prime}(n)\left[1-\frac{z}{p_{h}} h(n)+n^{\lambda_{\gamma} \lambda_{\alpha}}\left(\frac{p}{p_{h}}\right)^{-\lambda_{\gamma}}\right]+\lambda_{\gamma} \lambda_{\alpha} n^{\lambda_{\gamma} \lambda_{\alpha}-1}\left(\frac{p}{p_{h}}\right)^{-\lambda_{\gamma}} h(n)-\frac{c}{p_{h}}
$$

Using $h^{\prime}(n)=-h(n) \frac{k^{\prime}(n)}{k(n)}$, and substituting the expressions for $k^{\prime}(n)$ and $k(n)$, we obtain after rearranging terms

$$
\begin{gathered}
\frac{1}{p_{h}} \frac{\partial}{\partial n} \widetilde{\pi}(n)=-\frac{h(n)}{[k(n)]^{2}} \lambda_{\gamma} \lambda_{\alpha} n^{\lambda_{\gamma} \lambda_{\alpha}-1}\left(\frac{p}{p_{h}}\right)^{-\lambda_{\gamma}} p_{h} p_{h}^{\lambda_{\beta}}\left[1+n^{\lambda_{\gamma} \lambda_{\alpha}}\left(\frac{p}{p_{h}}\right)^{-\lambda_{\gamma}}\right]^{-\frac{\lambda_{\beta}}{\lambda_{\gamma}}} \\
\left\{\frac{z}{p_{h}} R\left[\frac{1}{p_{h}^{\lambda_{\beta}}\left[1+n^{\lambda_{\gamma} \lambda_{\alpha}}\left(\frac{p}{p_{h}}\right)^{-\lambda_{\gamma}}\right]^{-\frac{\lambda_{\beta}}{\lambda_{\gamma}}}}+\frac{\lambda_{\gamma}-\lambda_{\beta}}{\lambda_{\gamma}}\right]-\frac{\lambda_{\beta}}{\lambda_{\gamma}}\left[1+n^{\lambda_{\gamma} \lambda_{\alpha}}\left(\frac{p}{p_{h}}\right)^{-\lambda_{\gamma}}\right] k(n)\right\}-\frac{c}{p_{h}}
\end{gathered}
$$

The first term in curled brackets is negative and therefore a sufficient condition for $\frac{1}{p_{h}} \frac{\partial}{\partial n} \Pi(n)<0$ to hold is that

$$
\frac{h(n)}{k(n)} \lambda_{\alpha} \lambda_{\beta} n^{\lambda_{\gamma} \lambda_{\alpha}-1}\left(\frac{p}{p_{h}}\right)^{-\lambda_{\gamma}} p_{h} p_{h}^{\lambda_{\beta}}\left[1+n^{\lambda_{\gamma} \lambda_{\alpha}}\left(\frac{p}{p_{h}}\right)^{-\lambda_{\gamma}}\right]^{1-\frac{\lambda_{\beta}}{\lambda_{\gamma}}}<\frac{c}{p_{h}}
$$

Substituting the expression for $k(n)$ and rearranging terms this inequality reads

$$
\left[h(n) \lambda_{\alpha} \lambda_{\beta} n^{\lambda_{\gamma} \lambda_{\alpha}-1}\left(\frac{p}{p_{h}}\right)^{-\lambda_{\gamma}}-\frac{c}{p_{h}}\right] p_{h}^{\lambda_{\beta}}\left[1+n^{\lambda_{\gamma} \lambda_{\alpha}}\left(\frac{p}{p_{h}}\right)^{-\lambda_{\gamma}}\right]^{-\frac{\lambda_{\beta}}{\lambda_{\gamma}}}<\frac{c}{p_{h}}
$$


A sufficient condition for this inequality to hold is that

$$
\lambda_{\alpha} \lambda_{\beta} h(n) n^{\lambda_{\gamma} \lambda_{\alpha}-1}\left(\frac{p}{p_{h}}\right)^{-\lambda_{\gamma}}<\frac{c}{p_{h}}
$$

which, after using (17), simplifies to

$$
p x(n)<\frac{c}{\lambda_{\alpha} \lambda_{\beta}}
$$

Using Assumption 1, which implies that $\lambda_{\alpha} \lambda_{\beta}<1$, and the free entry condition (5) $p x(n)=c+s$, this inequality reads

$$
s<c \frac{1-\lambda_{\alpha} \lambda_{\beta}}{\lambda_{\alpha} \lambda_{\beta}}
$$

This last inequality is always satisfied for $s<0$ and therefore $\frac{\partial}{\partial n} \widetilde{\pi}(n)<0$ for all $s<0$ which implies that $s<0$ can never be optimal.

Proof. [Proof of Lemma 3] Rewriting $\Pi(n)$ using the relationship between $x(n)$ and $h(n)$ as in (17) we obtain

$$
\frac{\Pi(n)}{p_{h}}=h(n)\left[1-\frac{1}{2} \frac{z}{p_{h}} h(n)+n^{\lambda_{\gamma} \lambda_{\alpha}}\left(\frac{p}{p_{h}}\right)^{-\lambda_{\gamma}}\right]-\frac{c}{p_{h}} N
$$

Taking the first derivative with respect to $n$ we obtain

$$
\frac{1}{p_{h}} \frac{\partial}{\partial n} \Pi(n)=h^{\prime}(n)\left[1-\frac{z}{p_{h}} h(n)+n^{\lambda_{\gamma} \lambda_{\alpha}}\left(\frac{p}{p_{h}}\right)^{-\lambda_{\gamma}}\right]+\lambda_{\gamma} \lambda_{\alpha} n^{\lambda_{\gamma} \lambda_{\alpha}-1}\left(\frac{p}{p_{h}}\right)^{-\lambda_{\gamma}} h(n)
$$

which, after using $h^{\prime}(n)=-h(n) \frac{k^{\prime}(n)}{k(n)}$, can be written as

$$
\begin{aligned}
\frac{1}{p_{h}} \frac{\partial}{\partial n} \Pi(n)=- & \frac{h(n)}{k(n)}\left\{k^{\prime}(n)\left[1-\frac{z}{p_{h}} h(n)+n^{\lambda_{\gamma} \lambda_{\alpha}}\left(\frac{p}{p_{h}}\right)^{-\lambda_{\gamma}}\right]+\right. \\
& \left.-\lambda_{\gamma} \lambda_{\alpha} n^{\lambda_{\gamma} \lambda_{\alpha}-1}\left(\frac{p}{p_{h}}\right)^{-\lambda_{\gamma}} k(n)\right\}
\end{aligned}
$$

Substituting the expression for $k(n)$ and $k^{\prime}(n)$ and rearranging terms we obtain

$$
\begin{gathered}
\frac{\partial}{\partial n} \frac{\Pi(n)}{p_{h}}=-\frac{h(n)}{[k(n)]^{2}} \lambda_{\gamma} \lambda_{\alpha} n^{\lambda_{\gamma} \lambda_{\alpha}-1}\left(\frac{p}{p_{h}}\right)^{-\lambda_{\gamma}} p_{h} p_{h}^{\lambda_{\beta}}\left[1+n^{\lambda_{\gamma} \lambda_{\alpha}}\left(\frac{p}{p_{h}}\right)^{-\lambda_{\gamma}}\right]^{-\frac{\lambda_{\beta}}{\lambda_{\gamma}}} \\
\left\{\begin{array}{c}
-\frac{z}{p_{h}} R\left[\frac{1}{p_{h}^{\lambda_{\beta}}\left[1+n^{\lambda_{\gamma} \lambda_{\alpha}}\left(\frac{p}{p_{h}}\right)^{-\lambda_{\gamma}}\right]^{-\frac{\lambda_{\beta}}{\lambda_{\gamma}}}}+\frac{\lambda_{\gamma}-\lambda_{\beta}}{\lambda_{\gamma}}\right]+ \\
\left.-\frac{\lambda_{\beta}}{\lambda_{\gamma}}\left[1+n^{\lambda_{\gamma} \lambda_{\alpha}}\left(\frac{p}{p_{h}}\right)^{-\lambda_{\gamma}}\right] k(n)\right\}
\end{array}\right.
\end{gathered}
$$


where the term in the first line, multiplying the two terms in curled brackets, is positive; the first term in curled brackets (in the second line) is negative, decreasing in $n$, converges to zero in the limit of $n \rightarrow 0$ and to $-\frac{z}{p_{h}} R\left(p_{h}^{-\lambda_{\beta}}+\frac{\lambda_{\gamma}-\lambda_{\beta}}{\lambda_{\gamma}}\right)$ for $n \rightarrow \infty$; the second term in curled brackets (in the third line) is positive, decreasing in $n$, converges to $\infty$ for $n \rightarrow 0$ and, since $\lim _{n \rightarrow \infty} k(n)=$ $p_{h}\left(1+p_{h}^{\lambda_{\beta}}\right)$, it converges to $-\frac{\lambda_{\beta}}{\lambda_{\gamma}} p_{h}\left(1+p_{h}^{\lambda_{\beta}}\right)$ for $n \rightarrow \infty$. Consequently, a unique maximum exists if $\frac{z}{p_{h}} R\left(p_{h}^{-\lambda_{\beta}}+1-\frac{\lambda_{\beta}}{\lambda_{\gamma}}\right)>-\frac{\lambda_{\beta}}{\lambda_{\gamma}} p_{h}\left(1+p_{h}^{\lambda_{\beta}}\right)$, i.e. for $R \frac{z}{p_{h}}$ sufficiently large. Notice that, since

$$
-\frac{\lambda_{\beta}}{\lambda_{\gamma}} \frac{p_{h}\left(1+p_{h}^{\lambda_{\beta}}\right)}{\left(p_{h}^{-\lambda_{\beta}}+1-\frac{\lambda_{\beta}}{\lambda_{\gamma}}\right)}<p_{h}\left(1+p_{h}^{\lambda_{\beta}}\right)
$$

Assumption 1 guarantees that a unique maximum exists. Moreover, the profit function $\Pi(n)$ is locally concave at $n^{*}$.

Since $\frac{1}{p_{h}} \frac{\partial^{2}}{\partial z \partial n} \Pi(n)<0, \frac{\partial}{\partial z} n^{*}<0$ and therefore $\frac{\partial}{\partial z} s^{*}>0$

Proof. [Proof of Lemma 4] We show that, for $c=c^{e}$ and $n=n^{M},\left.\frac{\partial}{\partial n} \frac{\Pi(n)}{p_{h}}\right|_{n=n^{M}}<0$ and therefore $s^{*}>0$ follows. Using the relationship between $x(n)$ and $h(n)$ in (17), we can write profits as

$$
\frac{\Pi(n)}{p_{h}}=\pi(n)+h(n) n^{\lambda_{\gamma} \lambda_{\alpha}}\left(\frac{p}{p_{h}}\right)^{-\lambda_{\gamma}}-\frac{c}{p_{h}} N
$$

Taking the first derivative and evaluating the expression at $n=n^{M}$ and $c=c^{e}$, and taking into account that $\pi^{\prime}\left(n^{M}\right)=0$, we obtain

$$
\left.\frac{1}{p_{h}} \frac{\partial}{\partial n} \Pi(n)\right|_{n=n^{M}, c=c^{e}}=\left(\frac{p}{p_{h}}\right)^{-\lambda_{\gamma}}\left(n^{M}\right)^{\lambda_{\gamma} \lambda_{\alpha}}\left[h^{\prime}\left(n^{M}\right)+\lambda_{\gamma} \lambda_{\alpha} h\left(n^{M}\right)\left(n^{M}\right)^{-1}\right]
$$

or

$$
\left.\frac{1}{p_{h}} \frac{\partial}{\partial n} \Pi(n)\right|_{n=n^{M}, c=c^{e}}=\left(\frac{p}{p_{h}}\right)^{-\lambda_{\gamma}}\left(n^{M}\right)^{\lambda_{\gamma} \lambda_{\alpha}} h\left(n^{M}\right)\left(n^{M}\right)^{-1}\left[-\frac{k^{\prime}\left(n^{M}\right)}{k\left(n^{M}\right)} n^{M}+\lambda_{\gamma} \lambda_{\alpha}\right]
$$

Using $k^{\prime}\left(n^{M}\right)$ and $k(n)$, and rearranging terms we obtain that $\left.\frac{1}{p_{h}} \frac{\partial}{\partial n} \Pi(n)\right|_{n=n^{M}, c=c^{e}}<0$ if

$$
\left[-\frac{\lambda_{\beta}}{\lambda_{\gamma}}\left(n^{M}\right)^{\lambda_{\gamma} \lambda_{\alpha}}\left(\frac{p}{p_{h}}\right)^{-\lambda_{\gamma}}-1\right] p_{h}^{\lambda_{\beta}}\left[1+\left(n^{M}\right)^{\lambda_{\gamma} \lambda_{\alpha}}\left(\frac{p}{p_{h}}\right)^{-\lambda_{\gamma}}\right]^{-\frac{\lambda_{\beta}}{\lambda_{\gamma}}}<1
$$

Since the first term in squared brackets is decreasing in $n^{M}$ and since $n \geq 1$, a sufficient condition for 
the inequality to hold is that

$$
-\frac{\lambda_{\beta}}{\lambda_{\gamma}}\left(\frac{p}{p_{h}}\right)^{-\lambda_{\gamma}}<1
$$

which, because of Assumption 2, is always satisfied. By continuity, it follows that $s^{*}>0$ is optimal also for some values of $c>c^{e}$.

Proof. [Proof of Proposition 2] For $n^{*}<N, \frac{\partial}{\partial N} \widetilde{\Pi}<0$ and, using the envelope theorem, $\frac{\partial}{\partial c} \widetilde{\Pi}<0$. Consequently, for $N$ sufficiently large $\widetilde{\Pi}<\pi\left(n^{L}\right)$. Let us next characterize the threshold $\bar{N}$. Notice that we restrict the analysis to the case $c<c^{e}$, where $s^{*}>0$.

For $c<c^{e}, \frac{\partial}{\partial c} \pi\left(n^{L}\right)>0$, while $\frac{\partial}{\partial c} \widetilde{\Pi}<0$. As a consequence, $\bar{N}$ is decreasing in $c$.

Using the envelope theorem, we observe that $\frac{\partial}{\partial z} \widetilde{\Pi}<0$. For $c<c^{e}, \frac{\partial}{\partial z} \pi\left(n^{L}\right)=-\frac{1}{2} h\left(n^{L}\right)^{2}$, while $\frac{\partial}{\partial z} \widetilde{\Pi}=-\frac{1}{2} h\left(n^{M}\right)^{2}$. Since $h^{\prime}(n)>0, n^{M}<n^{L}$ and $n^{M}$ is increasing in $z,\left|\frac{\partial}{\partial z} \widetilde{\Pi}\right|<\left|\frac{\partial}{\partial z} \pi\left(n^{L}\right)\right|$, that is profits in the case of a domination strategy decrease at a lower pace than profits without a domination strategy. As a consequence $\bar{N}$ is increasing in $z$. 